\title{
Des Objectifs du Millénaire pour le Développement à réévaluer? Les impacts de la crise financière actuelle en Afrique subsaharienne
}

\author{
Marie Fall, Ph.D. et Salmata Ouedraogo, Ph.D. \\ Université du Québec à Chicoutimi
}

\section{INTRODUCTION}

Alors que jusqu'aux années 90, la réalisation du développement était le slogan des politiques et des actions des États africains, aujourd'hui, le thème de mobilisation a surtout trait à la lutte contre la pauvreté. La place centrale conférée à cette question dans les actions des États africains ainsi que dans les programmes des institutions internationales est un puissant révélateur de l'ampleur du phénomène de la pauvreté dans les pays africains. Elle illustre également l'adossement des priorités des États africains sur les agendas de la Banque mondiale et du Fonds monétaire international qui n'ont cessé de jouer un rôle prépondérant dans la formulation et la mise en œuvre des politiques macroéconomiques nationales.

\section{L'Afrique subsaharienne détient} aujourd'hui les taux de pauvreté les plus

élevés par continent. En 1999, près de 47 \%

de la population africaine vivaient avec moins d'un dollar par jour et étaient plongés dans une situation d'extrême pauvreté.

L'Afrique subsaharienne détient aujourd' hui les taux de pauvreté les plus élevés par continent. En 1999, près de $47 \%$ de la population africaine vivaient avec moins d'un dollar par jour et étaient plongés dans une situation d'extrême pauvreté. La proportion des personnes vivant avec moins de deux dollars par jour est demeurée élevée et se situe autour de $75 \%$ de la population. Le rapport de l'Organisation des Nations Unies sur les Objectifs du Millénaire pour le Développement de 2009 souligne que l'Afrique subsaharienne comptait, en 2005, 100 millions de personnes extrêmement pauvres de plus qu'en 1990. De plus, le taux de pauvreté restait au-dessus de $50 \%$. Même si les prévisions ${ }^{1}$ font état d'une diminution de la pauvreté monétaire d'ici 2015, les taux qui seront observés dans environ une décennie resteront tous aussi élevés. En effet, $40 \%$ des africains continueront de vivre avec moins d'un dollar par jour, alors que le pourcentage de ceux disposant de moins de deux dollars par jour sera de $68 \%$ en 2015. Devant ce constat, on est ainsi amené à reconnaître que quatre décennies de développement n'ont pas permis de juguler ni d'atténuer les effets les plus durs de la pauvreté. Bien au contraire, le phénomène a revêtu une ampleur et une acuité de plus en plus aiguës à la faveur de la crise économique et des programmes d'ajustement structurel, de l'instabilité politique, des perturbations environnementales et d'un contexte international peu favorable. La pauvreté dont il est question ici se décline donc en un manque ou un défaut d'accès à un certain nombre de biens et services indispensables pour la satisfaction des besoins jugés vitaux: se nourrir, se soigner, bénéficier d'un logement décent et disposer des revenus permettant de satisfaire ces besoins essentiels et ainsi de jouir pleinement de ses capacités.

La pauvreté dont il est question ici se décline donc en un manque ou un défaut d'accès à un certain nombre de biens et services indispensables pour la satisfaction des besoins jugés vitaux.

La massification de la pauvreté a contribué à placer l'Afrique au début des années 2000 au centre des préoccupations, des discours et des agendas des acteurs du monde du développement: États, organismes spécialisés des Nations Unies, bailleurs de fonds, institutions internationales, 
organisations non gouvernementales. La prise de conscience des proportions de la pauvreté a alors débouché sur l'élaboration des documents stratégiques de réduction de la pauvreté qui constituent, depuis lors, le cadre d'orientation des actions des États concernés et des interventions des partenaires au développement. Cette prise de conscience s'est matérialisée en septembre 2000 par l'adoption des Objectifs du Millénaire pour le Développement au cours d'un sommet des Nations Unies, par un vaste parterre de chefs d'État et de gouvernement qui se sont ainsi engagés à «faire de leur mieux pour éradiquer la pauvreté, défendre la dignité et l'égalité et parvenir à la paix, à la démocratie et assurer la durabilité des ressources environnementales ${ }^{2} »$.

Les Objectifs du Millénaire pour le Développement ont été établis suivant des scénarios optimistes et une répartition des responsabilités et des engagements qui en appelaient au devoir de solidarité des pays riches.

Des objectifs visionnaires ont été définis et devaient par conséquent libérer les pays en développement de la pauvreté extrême, de la faim, de l'analphabétisme et de la maladie, et que les enfants africains auront droit à une instruction de qualité et mèneront une vie saine et productive. Â cet effet, des cibles pour arriver à l'égalité des sexes et à l'autonomisation des femmes, à un environnement durable et à un partenariat mondial pour le développement ont été établis. Qu'en est-il aujourd'hui?

Les Objectifs du Millénaire pour le Développement ont été établis suivant des scénarios optimistes et une répartition des responsabilités et des engagements qui en appelaient au devoir de solidarité des pays riches. Ils reposent sur l'impérieuse nécessité de combattre l'extrême pauvreté. À la lumière de l'intensité de la crise actuelle et de l'ampleur de ses conséquences actuelles et anticipées, il est opportun d'examiner les incidences que la crise financière aura sur les Objectifs du Millénaire pour le Développement. En s'appuyant sur les données macroéconomiques disponibles dans les rapports et études de la Banque mondiale et du Fonds monétaire international ainsi que des institutions spécialisées des Nations Unies, le présent article analyse les répercussions de la crise économique en Afrique subsaharienne, examine les difficultés d'atteindre les Objectifs du Millénaire pour le Développement et propose une esquisse de scénarios pour le futur en matière de lutte contre la pauvreté.

\section{LES RÉPERCUSSIONS DE LA CRISE ÉCONOMIQUE EN AFRIQUE SUBSAHARIENNE}

En 2000, les dirigeants du monde ont dressé un plan pour un monde meilleur. De plus, ils se sont engagés à n'épargner aucuns efforts pour accomplir cette vision. Plusieurs objectifs de développement, comme les Objectifs du Millénaire pour le Développement ont été engagés au plan international. Cet engagement a fait croire que les populations échapperont aux fléaux tels que la pauvreté, la faim, la malnutrition, les problèmes de santé et l'analphabétisme.

Huit objectifs pour 2015 ont été adoptés par les pays du monde consistant à réduire l'extrême pauvreté et la faim, assurer l'éducation primaire pour tous, promouvoir l'égalité des sexes et l'autonomisation des femmes, réduire la mortalité infantile et améliorer la santé maternelle, combattre le VIH/SIDA et autres maladies mortelles, préserver l'environnement et mettre en place un partenariat mondial pour le développement.

Mais, aujourd'hui, selon les statistiques de la Banque mondiale, de nombreux pays, notamment en Afrique subsaharienne, sont loin d'atteindre les Objectifs du Millénaire pour le Développement. Ce triste constat a été fait bien avant la crise financière et économique actuelle. Et pire encore, avec la récente vague de crises, il sera encore plus difficile et coûteux de les atteindre (crise alimentaire, hausse du prix des matières premières, crise financière, crise économique). 


\section{La réalisation des Objectifs du Millénaire pour le Développement commence à \\ ralentir, voire à s'inverser, sachant qu'il reste moins de la moitié du chemin à parcourir avant la date butoir de 2015.}

Ainsi, la réalisation des Objectifs du Millénaire pour le Développement commence à ralentir, voire à s'inverser, sachant qu'il reste moins de la moitié du chemin à parcourir avant la date butoir de 2015. Tout d'abord, il est important de souligner que le processus d'intégration de l'Afrique dans l'économie mondiale, amorcée depuis environ une quinzaine d'années, a accru sa vulnérabilité aux chocs négatifs sur les flux financiers entrainant ainsi de graves conséquences sur la croissance économique des différents pays. Par exemple, les investissements directs étrangers ${ }^{2}$ ont baissé de près de $10 \%$ en 2008 (investissements directs étrangers, érosion des recettes d'exportations et baisse des fonds envoyés par les expatriés). De plus, les performances macroéconomiques de l'Afrique en 2008 ont été faibles $^{3}$. Le continent a affiché dans son ensemble une croissance du PIB de 5,7\%, contre $6,1 \%$ en 2007. Mais la crise économique mondiale devrait provoquer un tassement brutal en 2009 et ramener le taux de la croissance à $2,8 \%$ ces cinq dernières années. L'aggravation de la conjoncture économique mondiale pourrait encore assombrir les perspectives de croissance de l'Afrique pour 2009 comme pour 2010. Concernant l'inflation, la hausse du prix du pétrole, des engrais et des denrées alimentaires a largement aggravé les taux d'inflation dans les différents pays rendant la vie plus chère pour des millions de personnes. Dans son rapport annuel, le Bureau international du travail souligne que le nombre de chômeurs passerait à 30 millions en 2009 contre 18 millions en 2007. De plus, près de 200 millions de personnes, dont la majorité évolue dans des économies en développement, basculeraient dans l'extrême pauvreté.

\section{DES OBJECTIFS DU MILLÉNAIRE POUR LE DÉVELOPPEMENT DEVENUS HORS D'ATTEINTE?}

Les Objectifs du Millénaire pour le Développement ont été articulés autour de la lutte contre la pauvreté à travers une série d'agendas, d'échéances et de stratégies d'action. Ceux-ci sont orientés vers la disparition de l'extrême pauvreté et de la faim, la promotion de l'égalité des sexes et de l'autonomisation des femmes, l'amélioration de la santé des mères, la réduction de la mortalité infantile et des ravages des maladies, la promotion de la durabilité des ressources naturelles et, enfin, la mise en place d'un partenariat mondial pour le développement. Les Objectifs du Millénaire pour le Développement ont été adoptés dans un contexte économique international qui ne laissait en rien présager la crise financière qui secoue le monde depuis 2008. Les conséquences en sont déjà dramatiques et ont touché la vaste majorité des pays industrialisés et émergents. Le troisième bloc de pays victimes de ce «tsunami » financier est formé des pays en développement, ce qui est le cas de la majorité des pays d'Afrique subsaharienne.

\section{La troisième vague de pays victimes de ce « tsunami » financier est formée des pays en développement, ce qui est le cas de la majorité des pays d'Afrique subsaharienne.}

La crise financière et économique mondiale, la plus grave depuis celle de 1929, est devenue une crise humaine et de développement. Elle est apparue dans les pays développés et a rapidement et inexorablement gagné l'ensemble des pays en développement. Depuis le début de l'année 2009, la crise a atteint l'Afrique et ses impacts se lisent à travers la chute de la demande de matières premières, le reflux des investissements étrangers, le recul de l'aide internationale et la baisse des envois de fonds des travailleurs émigrés. La Banque mondiale prévoit que la crise économique actuelle y entraînera 550 millions de personnes dans l'extrême pauvreté, avec moins de 1,25 dollar de revenu par jour ${ }^{4}$. Certains analystes prévoient 
que la récession contribuera à faire mourir au cours de leur première année 700000 enfants africains. Les causes de cette surmortalité sont toutes imputables à la crise : nutrition dégradée, eau potable plus rare, assainissement plus inefficace, services de santé moins présents, parents obligés de délaisser leurs enfants pour assurer la subsistance de la famille. Deux constats majeurs s'imposent dans l'analyse des interrelations entre la crise financière et économique actuelle et l'atteinte des Objectifs du Millénaire pour le Développement.

\section{Le respect des engagements financiers pour la réalisation des Objectifs du Millénaire pour le Développement en Afrique subsaharienne est devenu aléatoire. Les pays donateurs, confrontés à leurs propres difficultés, peuvent être amenés à ne pas tenir leurs promesses en matière d'aide au développement et d'investissement.}

Premièrement, l'atteinte des Objectifs du Millénaire pour le Développement suppose un soutien financier important de la part des donateurs internationaux (pays développés, organismes d'aide au développement, institutions financières internationales) pour mettre en place les enveloppes budgétaires devant financer les programmes sociaux nécessaires à la réduction de la pauvreté et l'atteinte à la dignité humaine (adduction d'eau, infrastructures scolaires et de santé, programmes d'emploi). Or, placés dans une crise financière et économique dont la résolution requiert des moyens financiers colossaux comme en témoignent les volumes financiers mobilisés dans le cadre des plans de sauvetage des institutions bancaires et de relance économique des pays développés, le risque est grand pour que les engagements pris pour l'atteinte des Objectifs du Millénaire pour le Développement fassent les frais de la crise actuelle. L'aide bilatérale comme multilatérale au développement qui s'éloignaient déjà de plus en plus de la norme onusienne de $0,7 \%$ du revenu national brut des pays donateurs risquent de beaucoup pâtir de cette situation. Le respect des engagements financiers pour la réalisation des Objectifs du Millénaire pour le Développement en Afrique subsaharienne est devenu aléatoire. Les pays donateurs, confrontés à leurs propres difficultés, peuvent être amenés à ne pas tenir leurs promesses en matière d'aide au développement et d'investissement.

Deuxièmement, après plusieurs décennies de crise économique et d'austérité budgétaire, la majorité des pays africains ont renoué avec une croissance positive au cours des années 2000 grâce à la hausse significative des recettes d'exportation. Cette nouvelle donne rendait possible de consacrer un budget plus accru aux secteurs sociaux de base (éducation, santé, alimentation) au cœur des Objectifs du Millénaire pour le Développement. Avec la crise financière actuelle, l'éclaircie risque d'être éphémère. En effet, les prévisions de la Banque mondiale font état d'une chute de la croissance sur le continent de $6 \%$ en 2008 à $3 \%$ en 2009 avec des conséquences sociales désastreuses qui vont exacerber encore davantage le problème de la pauvreté. La réduction de moitié de la croissance réduit drastiquement l'amélioration du revenu par habitant, car la croissance démographique continue d'augmenter.

\section{Les prévisions de la Banque mondiale font état d'une chute de la croissance sur le continent de $6 \%$ en 2008 à $3 \%$ en 2009 avec des conséquences désastreuses au plan social qui vont exacerber encore davantage le problème de la pauvreté.}

L'Afrique subsaharienne, nullement responsable de la crise financière et économique, est directement touchée par les répercussions. Plusieurs facteurs expliquent cette situation: crise mondiale, pressions sur les ressources, réduction considérable de la propension à consommer dans les pays développés accompagnés de politiques d'incitation à l'achat de produits locaux, actions qui se traduisent par une baisse des flux de capitaux vers les économies des pays en développement, baisse de la demande de matières premières dans les pays africains qui réduit les recettes fiscales et la capacité des États à faire face aux Objectifs du Millénaire pour le Développement. Les flux de capitaux provenant des immigrés constituent une source importante de revenus pour les populations mais aussi un 
paravent contre la paupérisation. La crise actuelle, en occasionnant une baisse sensible de ces transferts, ne fait qu'assombrir l'horizon eu égard à cette préoccupation développement.

À l'opposé des pays développés et des pays émergents qui ont pu mobiliser en un temps record plusieurs centaines de milliards de dollars au profit de leurs citoyens et de leurs entreprises, les pays africains, largement dépendants des flux des capitaux privés, peinent à se relever, même en tant que victimes collatérales.

\section{RÉÉVALUER OU RENFORCER LES OBJECTIFS DU MILLÉNAIRE POUR LE DÉVELOPPEMENT : ESQUISSE DE SCÉNARIOS}

De manière relative, la crise financière qui secoue aujourd'hui le monde, aussi grande soit elle, ne peut être considérée comme un obstacle à la satisfaction des besoins de base auxquels s'attaquent en grande partie les Objectifs du Millénaire pour le Développement. En effet, la crise actuelle n'a pas encore menacé les indices fondamentaux des pays développés par rapport à la satisfaction des besoins de nécessité ainsi qu'à l'accès aux services essentiels. Par conséquent, elle menace moins les aspects fondamentaux des Objectifs du Millénaire pour le Développement. Dans les pays défavorisés, le niveau des indices de développement est non seulement inquiétant mais aussi négativement sensible à la crise financière.

\section{De manière relative, la crise financière qui secoue aujourd'hui le monde, aussi grande soit elle, ne peut être considérée comme un obstacle à la satisfaction des besoins de base auxquels s'attaquent en grande partie les Objectifs du Millénaire pour le Développement.}

Cette crise, apparue aux États-Unis à la suite de l'explosion de la bulle immobilière, s'est vite propagée dans le reste du monde en raison des liens étroits qui existent entre les économies dans un cadre mondialisé. Une telle situation fait craindre le pire : un manque d'intérêt ainsi qu'un effritement de la volonté des pays développés face aux problèmes de pauvreté semblent prendre forme. Aujourd'hui, le secteur bancaire s'active à un rapatriement des fonds de leurs filiales mères. Selon le $\mathrm{FMI}^{5}$, cette menace est d'autant plus importante que les banques sont dotées de portefeuilles concentrés. D'où la nécessité de surveiller le système bancaire et de minimiser de telles pratiques en vue de réduire leurs effets néfastes.

D'un autre coté, se trouvent des économies en développement incapables de constituer des réserves ou de mettre sur pied des mécanismes de financement face à de tels impondérables. Elles se trouvent de toute évidence face à une crise dont elles commencent à peine à percevoir les méfaits. Au moment où les pays développés ont fini de satisfaire les besoins de base et constituent des réserves, les économies fragiles ne disposent pas de mécanismes ni d'automatismes qui leur permettent de réagir. Ce cadre fait craindre une accumulation d'arriérées ainsi qu'un endettement accru des pays en développement à la suite des baisses de trésorerie des gouvernements pouvant ainsi anéantir totalement ou en partie les efforts engagés dans le cadre de la mise en œuvre des documents stratégiques de réduction de la pauvreté.

La mise en œuvre des recommandations prenait en charge la réduction voir l'annulation des dettes dans certains pays en développement pour leur permettre d'orienter leurs ressources vers la satisfaction de besoins de base, la réduction de la pauvreté ainsi que l'atteinte des autres Objectifs du Millénaire pour le Développement. Les multiples effets collatéraux causés par la crise actuelle dans les pays en développement présagent de problèmes de trésorerie en raison des baisses importantes de ressources. Ces effets sont davantage prononcés dans des pays où la dépendance économique vis-à-vis de l'extérieur est importante. C'est le cas des pays exportateurs de produits de rente, des pays dans lesquels le système financier n'est pas diversifié, 
des pays qui comptent sur les envois de fonds des immigrés affectés dans leurs pays de résidence par la crise financière.

Cette situation fait aussi craindre la possibilité pour certains États africains de recourir, comme c'était le cas durant les années 80 , à des mécanismes ayant déjà montré leurs limites. Sur ce point, les privatisations à outrance ne font que fragiliser les économies en développement en augmentant leur dépendance vis-à-vis de l'extérieur. En général, les privatisations constituent des occasions d'acquisitions par des firmes étrangères de sociétés nationales.

\section{Les multiples effets collatéraux causés par la crise dans les pays en développement présagent de problèmes de trésorerie en raison des baisses importantes de ressources. Ces effets sont davantage prononcés dans des pays où la dépendance économique vis-à-vis de l'extérieur est importante.}

Compte tenu de ces constats, il est par conséquent nécessaire de se rendre à l'évidence. Bien avant la crise financière, les observateurs ont déjà souligné leur inquiétude par rapport au rythme dans lequel les réalisations des Objectifs du Millénaire pour le Développement avançaient. À la suite de l'observation des effets actuels ainsi que des sombres prévisions par rapport aux cibles fixés, les pays d'Afrique subsaharienne occupent les positions les plus délicates.

Malgré les difficultés, des avancées importantes ont été réalisées dans certains pays et corrélativement à des critères spécifiques. C'est la raison pour laquelle quelques experts montrent un certain optimisme par rapport à la capacité des acteurs d'aujourd'hui à atteindre les Objectifs du Millénaire pour le Développement en 2015. Cet optimisme est fondé en partie sur quelques résultats encourageant: les avancées notées au Malawi concernant les maladies infantiles, l'accès accru aux études primaires en Tanzanie et l'accès croissant à l'eau potable au Sénégal. Sous des conditions qui ont été énumérées dans la première partie et les réalités du terrain, les observations nous amènent à proposer certaines pistes. Sur ce point, plusieurs options peuvent s'offrir en s'inspirant des réflexions faites par des groupes d'experts depuis cette période de crise.

L'analyse des mécanismes par lesquels cette crise entre les pays africains et l'examen des impacts qu'elle peut engendrer porte à croire que la réévaluation devrait être moins axée sur la révision des objectifs, lesquels constituent une nécessité de justice sociale, que sur les stratégies à déployer en vue de se rapprocher au grand maximum des buts fixés.

Diverses pistes méritent ainsi d'être envisagées. D'abord, l'élargissement du champ de financement s'avère une condition essentielle. Le manque de financement des Objectifs du Millénaire pour le Développement a retenu l'attention des experts ${ }^{6}$, qui n'a pas manqué de mettre l'accent sur l'efficacité qui doit être observée dans la sélection des nouvelles ressources ainsi que leur opérationnalité à travers la rapidité de mobilisation et la faisabilité politique. À la lecture des positions prises par le Secrétaire Général des Nations Unies, l'urgence est à la recherche et au renforcement de nouveaux financements. Le maintien de la pression sur les donateurs doit aussi être soutenu, car le pourcentage de l'aide publique au développement ne représente pas grand-chose face aux montants débloqués pour surmonter cette crise.

Les États en développement doivent également déployer un effort interne pour mobiliser des ressources. En effet, étant donné la vulnérabilité de ces pays face à la crise financière, ces derniers devraient prendre conscience des effets possibles sur les apports extérieurs de fonds et rationaliser leurs choix budgétaires de manière à optimiser leurs dépenses dans le sens d'une meilleure redistribution des richesses.

Il faut aussi accroître la volonté des États, des bailleurs et autres acteurs qui interviennent dans l'atteinte des Objectifs du Millénaire pour le Développement. En effet, bien qu'elle soit salutaire, cette volonté doit davantage être renforcée pour notamment accroître le rythme de progression vers un monde plus juste et meilleur. 
Enfin, la dépendance par rapport à l'extérieur doit être réduite, surtout concernant les produits de consommation importés des pays occidentaux

\section{CONCLUSION}

La crise financière et économique actuelle, bien qu'étrangère à l'Afrique subsaharienne du point de vue de ses origines, ne manque pas d'avoir des effets sur les Objectifs du Millénaire pour le Développement. Cette situation amène, à juste titre, à s'interroger sur la nécessité ou pas de revoir ces derniers. Elle a engendré plusieurs effets visibles qui ne vont pas dans le sens de faciliter l'atteinte de ces objectifs en occasionnant des craintes liées notamment au manque de volonté, à l'accumulation d'arriérées, au problème de trésorerie, de licenciements, de privatisation et de retour au point de départ eu égard aux Objectifs du Millénaire pour le Développement.

Malgré l'enthousiasme affiché par certains groupes d'experts ayant réfléchi sur la question, il n'en demeure pas moins nécessaire de répondre à cette situation en posant des actes allant dans le sens de renforcer les acquis et d'augmenter le rythme de progression vers les buts visés. À la suite de cette réflexion, les propositions formulées suggèrent le renforcement de la pression sur les donateurs qui devront non seulement consolider mais aussi améliorer la valeur réelle de l'aide au développement. Des actions ciblées doivent aller dans le sens d'une réduction des fuites de capitaux des firmes étrangères vers les filiales mères, d'une augmentation en interne de l'effort des États dans la gouvernance, d'un élargissement du champs de financement externe et la création de nouveaux outils financiers susceptibles de rendre possible un refinancement des projets compromis par la crise financière.

La grandeur de la vision ainsi que les résultats combien encourageants observés jusque-là, doivent constituer des arguments suffisants pour ne pas verser dans le pessimisme. L'atteinte des Objectifs du Millénaire pour le Développement est possible grâce à des efforts misant sur l'efficacité des stratégies et des moyens. et asiatiques. En effet, l'Afrique fait face à une crise de dépendance dont l'ampleur influence la gravité du choc et des effets de la crise.

\section{Bibliographie}

${ }^{1}$ Banque mondiale (2003). « Rapport sur le développement dans le monde. Développement durable dans un monde dynamique : améliorer les institutions, la croissance, la qualité de vie », Washington.

2 PNUD (2006). «Au-delà de la pénurie : pouvoir, pauvreté et crise mondiale de l'eau. New York, Programme des Nations Unies pour le développement », Rapport mondial sur le développement humain 2006.

${ }^{3}$ OCDE (2009). «Perspectives économiques en Afrique $2009 »$.

${ }^{4}$ Banque mondiale (2009). «G-20 - Les pays en développement face à la crise », Le Groupe Banque mondiale.

5 Etchebarne-Bourdin, A. (2009). «Rapport 2008 sur les Objectifs du Millénaire pour le Développement », FMI-ONU.

${ }^{6}$ Reisen H. (2004). «Financer les Objectifs du Millénaire pour le Développement : Nouvelles approches », Organisation de Coopération et de Développement Économique. 


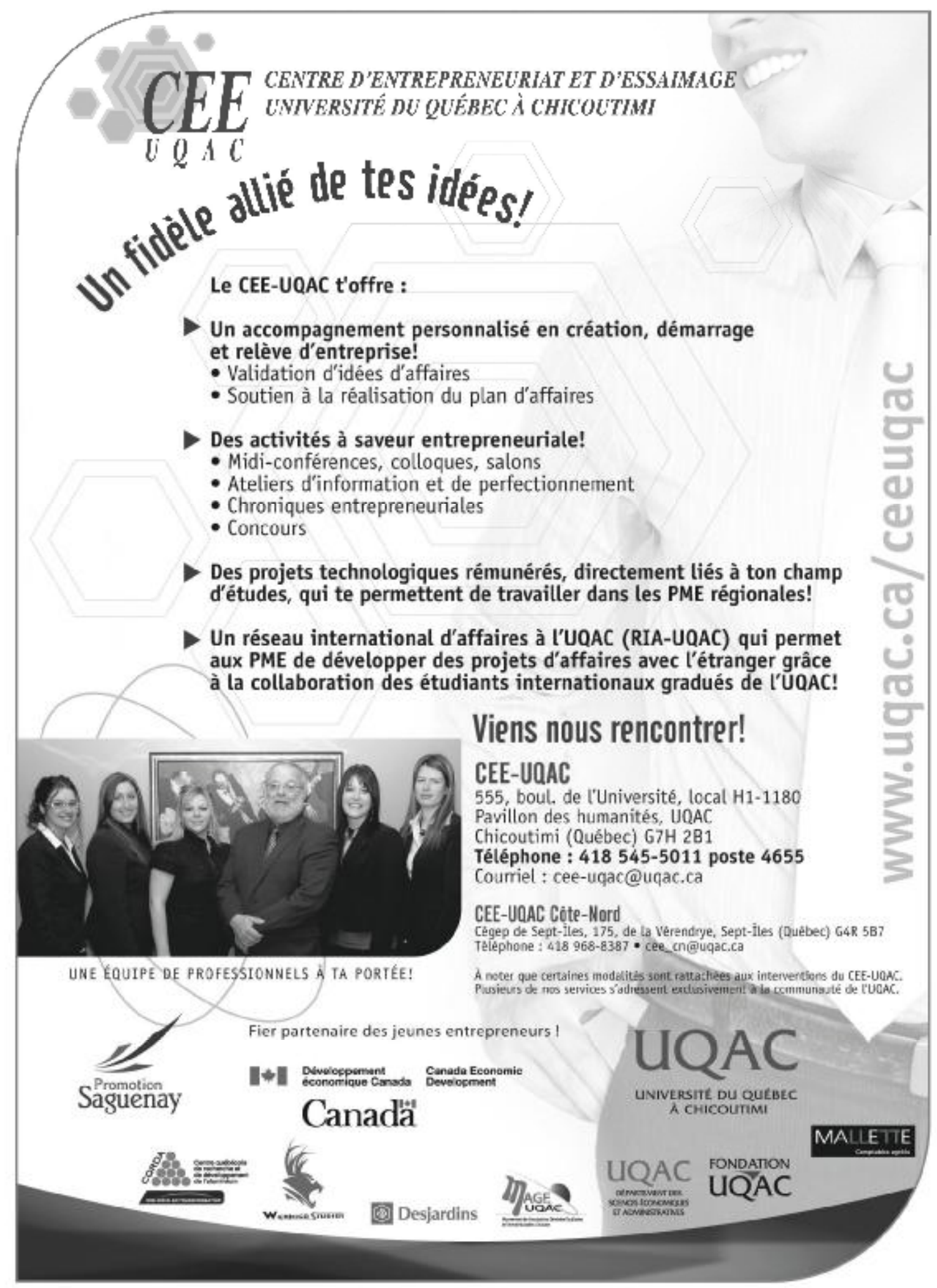

